\title{
Inhibition of prostate cancer cell growth in vivo with short hairpin RNA targeting SATB1
}

\author{
QIANG WANG $^{1 *}$, SHI-CHENG HU ${ }^{2 *}$, CHUN-SHENG YANG ${ }^{3 *}$, JIA-CUN CHEN ${ }^{2}$, \\ JUN-NIAN ZHENG ${ }^{4}$, XIAO-QING SUN $^{2}$ and JUN-QI WANG ${ }^{2}$
}

\begin{abstract}
${ }^{1}$ The First Clinical Medical College, Nanjing University of Chinese Medicine, Nanjing, Jiangsu 210046; ${ }^{2}$ Department of Urology, The Affiliated Hospital of Xuzhou Medical College, Xuzhou, Jiangsu 221002; ${ }^{3}$ Department of Dermatology, Affiliated Huai'an Hospital of Xuzhou Medical University, The Second People's Hospital of Huai'an, Huai'an 223002;

${ }^{4}$ Center of Clinical Oncology, The Affiliated Hospital of Xuzhou Medical College, Xuzhou, Jiangsu 221002, P.R. China
\end{abstract}

Received December 15, 2015; Accepted May 16, 2017

DOI: $10.3892 / \mathrm{ol} .2017 .7006$

\begin{abstract}
Despite previous advances, the treatment options for prostate cancer remain limited. For the purposes of gene knockdown, the utility of RNA interference has been demonstrated and is considered to have therapeutic potential. In the present study, a short hairpin RNA (shRNA) was used to assess the effect of special AT-rich sequence binding protein (SATB1) downregulation on the growth and metastatic potential of prostate cancer in xenograft nude mice. A plasmid carrying shRNA targeting SATB1, pSilencer-SATB1-shRNA, was successfully engineered. Using this plasmid, significant downregulation of SATB1 mRNA and protein expression in the DU145 prostate cancer cells was observed. pSilencer-SATB1-shRNA was demonstrated to be markedly efficacious against prostate cancer xenografts in nude mice. These results may lead to a novel method of improving gene therapy efficacy against prostate cancer via regulating the function of SATB1.
\end{abstract}

\section{Introduction}

In males over 50 years of age, prostate cancer is the most common form of malignancy in industrialized countries (1). In the United States of America (USA), it is the most commonly diagnosed type of cancer and the second most common cause of cancer-associated mortality (1). Annually, 23,000 males in the USA are diagnosed with the condition and almost 30,000 succumb to the disease (1). In its early stages, prostate cancer

Correspondence to: Professor Jun-Qi Wang, Department of Urology, The Affiliated Hospital of Xuzhou Medical College, 99 Huaihai Road West, Xuzhou, Jiangsu 221002, P.R. China

E-mail: a5716589@163.com

${ }^{*}$ Contributed equally

Key words: special AT-rich sequence binding protein 1, prostate cancer, short hairpin RNA, apoptosis may be treated through androgen depletion, radiation or chemotherapy (2). However, the disease is often problematic to control long term; $20-25 \%$ of those affected will eventually become androgen-independent and the disease will metastasize; advanced stage prostate cancer is incurable. In addition, the side effects of current treatment approaches mandate innovative and improved strategies to treat patients with prostate cancer.

One promising approach is gene therapy (3-5). In contrast to more conventional modalities, it may be tailored to the individual malignancy $(4,6)$. RNA interference (RNAi) is a method of utilizing post-transcriptional gene silencing, and has been demonstrated to be effective for gene knockdown; it appears to exhibit marked potential for patient therapy (7-9).

In prior studies, there was a focus on the transfection of synthetic small interfering RNA (siRNA) or plasmids in cancer cell research in order to induce the expression of short hairpin RNAs (shRNAs), making use of RNA polymerase III promoters $(8,10,11)$. A number of studies have investigated the silencing of specific cancer genes by RNAi in cell lines from various tissues types, resulting in a significant inhibition of cancer cell growth (9). In addition, this group has successfully used this knockdown technology to inhibit the expression of $\mathrm{Ki}-67$ in renal cancer cell lines.

The nuclear factor special AT-rich sequence binding protein 1 (SATB1) is the global chromatin organizer whose function is to regulate the structure of chromatin and gene expression (12). Its expression is abnormal in a number of cancer types. It has been suggested that SATB1 is an oncogene that promotes malignancy $(13,14)$. It has previously been suggested that it may be overexpressed in metastatic prostatic cancer, and its ability to promote cancer cell growth and invasion has been demonstrated (15). The present study reports the production of a novel plasmid containing shRNA against SATB1, pSilencer-SATB1-shRNA, and aimed to determine its antitumor efficacy in xenograft prostate tumors in nude mice.

\section{Materials and methods}

Cell lines and vectors. The DU145 prostate cancer cell line was obtained from the Cell Bank of Type Culture Collection of 
Chinese Academy of Sciences (Shanghai, China), and cultured in RPMI-1640 medium (Gibco; Thermo Fisher Scientific, Inc., Waltham, MA, USA), to which $10 \%$ heat-inactivated fetal bovine serum (Gibco; Thermo Fisher Scientific, Inc.), 4 mM glutamine, $50 \mathrm{U} / \mathrm{ml}$ penicillin and $50 \mu \mathrm{g} / \mathrm{ml}$ streptomycin had been added. The culture was performed at $37^{\circ} \mathrm{C}$ in a humidified atmosphere with $5 \% \mathrm{CO}_{2}$. The cells were routinely screened to verify they were free of mycoplasma contamination, and were used for experiments in the logarithmic phase of growth. The pSilencer-SATB1-shRNA plasmid and pSilencer plasmid were kindly provided by Professor Li Jun Mao. pSilencer plasmids were dissolved in K-PBS medium from KeyGen Biotech Co., Ltd (Nanjing, China) and used at concentrations of $1 \mathrm{~g} / \mathrm{l}$.

Xenograft tumor model in nude mice. The Shanghai Experimental Animal Center of the Chinese Academy of Sciences (Shanghai, China) provided 24 (4-5 week-old ) male BALB/c nude mice weighing $20 \mathrm{~g}$. Prior to implantation of the tumor cells, all mice were quarantined under the condition of constant temperature and humidity for 1 week. Mice had ad libitum access to food and water. Animal welfare and experimental procedures were carried out strictly in accordance with the Guide for the Care and Use of Laboratory Animals. A total of $2 \times 10^{6}$ DU145 cells was intraperitoneally injected into the right flank of the mice to establish the xenograft tumor model. The present study was approved by the Ethics Committee of The Affiliated Hospital of Xuzhou Medical University (Xuzhou, China).

Once the tumors reached a volume of $100-150 \mathrm{~mm}^{3}$, the mice were randomly divided into three groups, with 8 mice in each group. By group, the tumors were subsequently injected with $0.1 \mathrm{ml}$ pSilencer vector or pSilencer-SATB-1-shRNA for three consecutive days, or with $0.1 \mathrm{ml}$ PBS as a control. Over the following 30 days, calipers were used weekly to assess the size of the tumors. The volume of each tumor was then determined according to the following formula: Volume $\left(\mathrm{mm}^{3}\right)=$ length $\mathrm{x}$ width ${ }^{2} \mathrm{x} 1 / 2$. Data obtained for each group of 8 mice were expressed as the mean \pm standard error of the mean and assessed for statistical significance. BALB/c nude mice ( 8 per group) were sacrificed after 30 days by cervical dislocation, and the tumors were removed and fixed in $10 \%$ neutral formalin at room temperature for $24 \mathrm{~h}$ and embedded in paraffin for hematoxylin and eosin (H\&E) staining and immunohistochemistry.

Immunohistochemical staining. The harvested tumors were fixed in $10 \%$ formalin for $24 \mathrm{~h}$ at room temperature, embedded in paraffin and cut into 4-mm sections. Subsequent to the samples being deparaffinized with dimethylbenzene and rehydrated using a graduated alcohol series, endogenous peroxidase activity was blocked using $3 \% \mathrm{H}_{2} \mathrm{O}_{2}$ for $10 \mathrm{~min}$ at room temperature, and the sections were then incubated for $30 \mathrm{~min}$ at room temperature with goat serum (Beijing Solarbio Science \& Technology Co., Ltd., Beijing, China) to block membranes.

Immunohistochemistry was performed using an anti-SATB1 antibody (cat. no. 611182; GenHunter Corporation, Nashville, TN, USA). Following incubation with an anti-mouse secondary antibody (cat. no. PA174460; dilution, 1:1,000; Abcam Company ) at $37^{\circ} \mathrm{C}$ for $30 \mathrm{~min}$, SATB1
Table I. Calculated tumor volume in each group at 1, 5, 9, 13, 17,21 and 25 days.

\begin{tabular}{|c|c|c|c|}
\hline Days & $\begin{array}{l}\text { PBS } \\
\text { group }\end{array}$ & $\begin{array}{l}\text { pSilencer } \\
\text { group }\end{array}$ & $\begin{array}{c}\text { pSilencer- } \\
\text { SATB1-shRNA } \\
\text { group }\end{array}$ \\
\hline 1 & $96.42 \pm 12.04$ & $98.64 \pm 11.53$ & $100.31 \pm 13.25$ \\
\hline 5 & $682.34 \pm 198.02$ & $593.31 \pm 184.56$ & $508.90 \pm 139.78$ \\
\hline 9 & $1,248.23 \pm 185.73$ & $1,129.39 \pm 180.36$ & $1,045.32 \pm 189.56$ \\
\hline 13 & $1,528.34 \pm 190.13$ & $1,498.32 \pm 179.38$ & $1,228.23 \pm 179.83$ \\
\hline 17 & $1,923.37 \pm 281.04$ & $1,837.57 \pm 174.48$ & $1,148.78 \pm 137.45$ \\
\hline 21 & $2,310.98 \pm 202.56$ & $2,194.67 \pm 187.12$ & $703.16 \pm 110.34$ \\
\hline 25 & $2,713.38 \pm 276.99$ & $2,397.39 \pm 178.63$ & $519.03 \pm 110.83$ \\
\hline
\end{tabular}

Data are expressed as the means of tumor volume \pm standard deviation. The pSilencer-SATB1-shRNA vs. pSilencer, or PBS-treated groups $\left({ }^{\mathrm{a}} \mathrm{P}<0.05, \mathrm{n}=6\right)$. SATB1, special AT-rich sequence binding protein 1; sh, short hairpin RNA; pSilencer, plasmid silencer.

Table II. Tumor volume and inhibitory rate in each treatment group.

\begin{tabular}{lcc}
\hline Groups & $\begin{array}{c}\text { Tumor volume } \\
\left(\mathrm{mm}^{3}\right)\end{array}$ & $\begin{array}{c}\text { Tumor inhibitory } \\
\text { rate, } \%\end{array}$ \\
\hline PBS group (A) & $2713.38 \pm 276.99$ & - \\
pSilencer group (B) & $2397.39 \pm 178.63$ & $9.56 \pm 4.89$ \\
pSilencer-SATB-1- & a519.03 \pm 110.83 & $83.98 \pm 2.32$ \\
shRNA group (C) & & \\
\hline
\end{tabular}

Data are expressed as the means of tumor volume or inhibitory rate \pm standard deviation. ${ }^{a} \mathrm{P}<0.05, \mathrm{n}=6$. SATB 1 , special AT-rich sequence binding protein 1 ; sh, short hairpin; pSilencer, plasmid silencer.

expression was determined using 3,3'-diaminobenzidine (DAB; Sigma-Aldrich; Merck KGaA, Darmstadt, Germany) and enhanced using an Avidin-Biotin Reaction ABC kit (Vector Laboratories, Inc., Burlingame, CA, USA). The negative controls were tissue sections that had been stained without the primary antibody. The proportion of cells positive for SATB1 was determined using optical microscope by counting a minimum of 200 cells from 6 distinct areas, and then calculating the mean.

$H \& E$ staining and the terminal dUTP nick-end labelling (TUNEL) assay. Tumors were harvested and fixed in $4 \%$ paraformaldehyde for 30-50 min at room temperature, embedded in paraffin and cut into 4-mm sections. For histopathological analysis, the paraffin-embedded tissue sections were stained with H\&E at room temperature for 10-30 min. Following deparaffinization with xylene and rehydration using a graded alcohol series, apoptotic cells in the tumor tissue sections were quantified using the in situ Annexin V-FITC/PI Apoptosis Detection kit (Roche Diagnostics, Indianapolis, IN, USA). Following deparaffinization in xylene, sections 
A

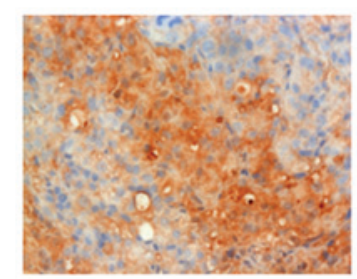

PBS
SATB1

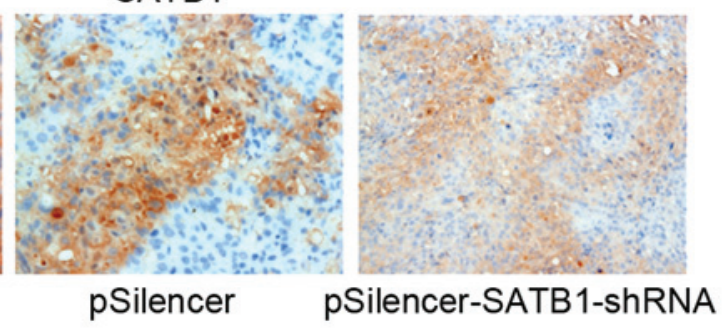

B

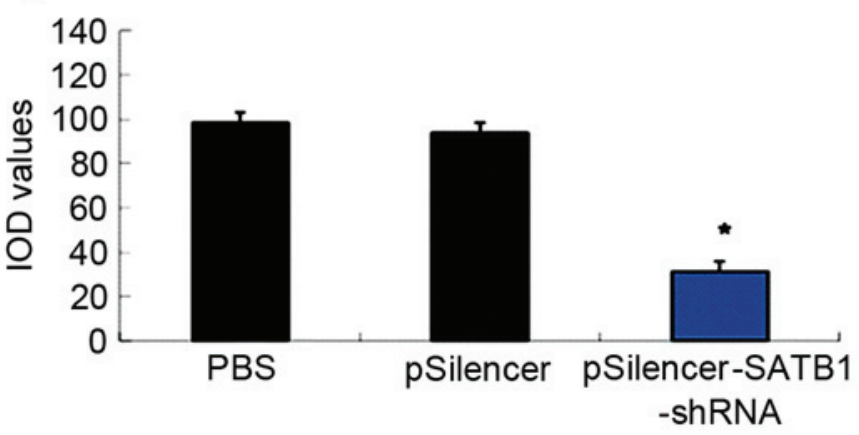

Figure 1. Immunohistochemical staining for SATB1 protein expression. (A) SATB1 protein is primarily located in the cytoplasm and cell membrane, dyed brown (magnification, x400). (B) Comparison of the IOD values in each treatment group. The pSilencer-SATB1-shRNA vs. pSilencer, or PBS-treated groups $\left({ }^{*} \mathrm{P}<0.05, \mathrm{n}=6\right)$. SATB1, special AT-rich sequence binding protein 1; IOD, integrated optical density; sh, short hairpin; pSilencer, plasmid silencer.

were rinsed twice with PBS and permeabilized using $15 \mu \mathrm{g} / \mathrm{ml}$ proteinase K (Yeasen, Shanghai, China) in $10 \mathrm{mM}$ Tris/ $\mathrm{HCl}$, $\mathrm{pH}$ 7.4-8.0 for $15 \mathrm{~min}$ at room temperature. Endogenous peroxidase activity was blocked using $3 \% \mathrm{H}_{2} \mathrm{O}_{2}$ for $10 \mathrm{~min}$ at room temperature. The sections were then incubated with an equilibration buffer and a terminal deoxynucleotidyl transferase enzyme, and then with an anti-digoxigenin-peroxidase conjugate. Negative controls were prepared by omitting the terminal transferase. Incubation with $10 \mathrm{mg} / \mathrm{ml} \mathrm{DAB}$ (Sigma-Aldrich; Merck KGaA, Darmstadt, Germany) for $30 \mathrm{~min}$ at room temperature was used to visualize peroxidase activity. Under light microscopy (magnification, x400), 6 fields were randomly selected from each sample and 100 cells were randomly selected from each field. The apoptotic rate was determined using the following formula: Number of apoptotic cells/100x100\%.

Western blot analysis. The cells were harvested from the plates, then the protein concentration was calculated by BCA method, and buffers containing 100ug protein were prepared per lane. Aliquots of the cell extracts were separated by $12 \%$ SDS-PAGE. The proteins were then transferred onto a nitrocellulose membrane. The membrane was then incubated overnight at $4^{\circ} \mathrm{C}$ with the following rabbit polyclonal antibodies: Anti-SATB1 (cat. no. 611182; GenHunter Corporation, Nashville, TN, USA), anti-MMP2 (cat. no. 2270S; Cell Signaling Technology, Inc., Danvers, MA, USA) and anti- $\beta$-actin (cat. no. sc-47778; Santa Cruz Biotechnology, Inc., Dallas, TX, USA). The membrane was then washed and incubated with alkaline phosphatase-conjugated secondary antibodies in TBS containing Tween-20 for $2 \mathrm{~h}$ at room temperature, and developed using the NBT/BCIP color substrate (Promega Corporation, Madison, WI, USA). The densities of the bands on the membrane were scanned and analyzed with Image 1.48 (National Institutes of Health, Bethesda, MD, USA) and LabWorks (UVP LLC, Upland, CA, USA).

Statistical analysis. Data are expressed as the mean \pm standard deviation and analyzed using one-way analysis of variance followed by Duncan's new multiple range test or the Newman-Keuls test as appropriate by statistical software (SPSS Base 15.0 for Windows, SPSS Inc., Chicago, IL, USA). $\mathrm{P}<0.05$ was considered to indicate a statistically significant difference.

\section{Results}

SATB1 protein expression in tumor tissues. The 8 control mice injected with PBS were designated as group A, the 8 mice injected with the empty pSilencer vector as group B and the 8 mice injected with the pSilencer-SATB1-shRNA as group C. The expression of SATB1 protein in tumor tissue samples obtained from each of the three groups was detected using immunohistochemical staining and western blot analysis. Immunohistochemical staining demonstrated that the SATB1 protein was expressed primarily in the cytoplasm and the cell membrane (brown staining, Fig. 1A). By using the ImageJ analysis software, the integrated optical density (IOD) absorbance values for PBS, pSilencer, and pSilencer-SATB1-shRNA were $99.86 \pm 2.58,93.83 \pm 2.15$ and $32.78 \pm 0.53$, respectively. Compared with the other two groups, the SATB1 protein expression, based on the IOD, in the pSilencer-SATB1-shRNA group was significantly low $(\mathrm{P}<0.05$; Fig. 1B). Additionally, western blot analysis demonstrated that the proportion of SATB1 protein in group 1, group 2 and group 3 was 98.6 $\pm 5.73,95.73 \pm 4.52$ and $31.4 \pm 5.78 \%$, respectively (Fig. 2). Based on the data from the western blot analysis, the SATB1 protein expression in the pSilencer-SATB1-shRNA group was significantly low when compared with the two control groups $(\mathrm{P}<0.05$; Fig. 2). 
A
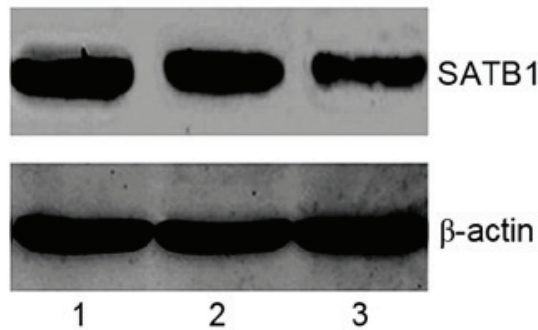

B

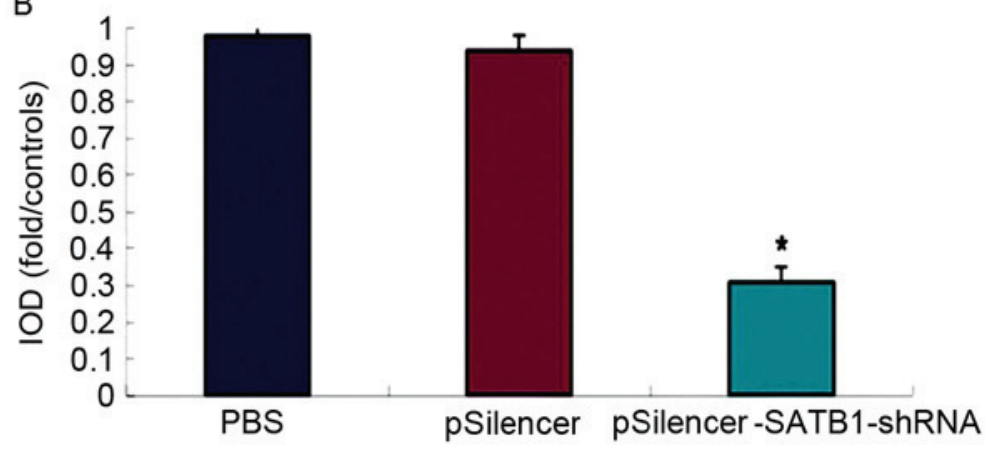

Figure 2. Western blot analysis of STAT1 protein expression. (A) Lane 1, PBC, lane 2 pSilencer; lane 3, pSilencer-SATB1-shRNA (B) Semi-quantification of western blot analysis demonstrating the level of SATB1 expression vs. the $\beta$-actin control. "P<0.05 vs. pSilencer and PBS-treated groups, $\mathrm{n}=6$. SATB1, special AT-rich sequence binding protein 1; sh, short hairpin; pSilencer, plasmid silencer.

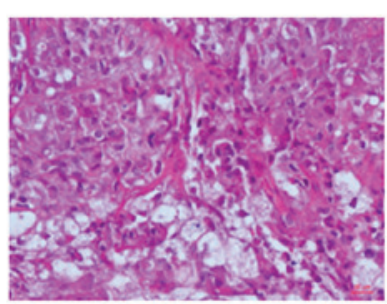

PBS

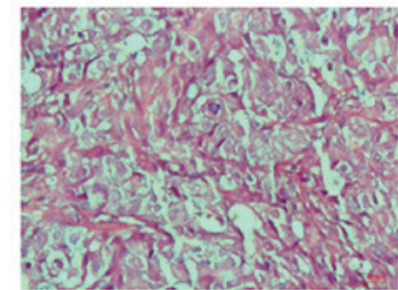

pSilencer

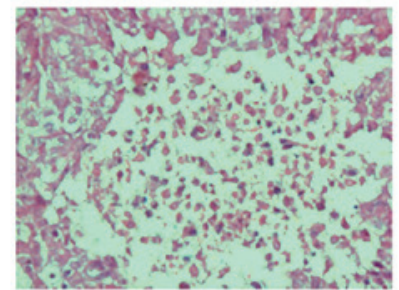

pSilencer-SATB1-shRNA

Figure 3. Hematoxylin and eosin staining of tumor tissue samples. SATB1, special AT-rich sequence binding protein 1; sh, short hairpin; pSilencer, plasmid silencer.

A

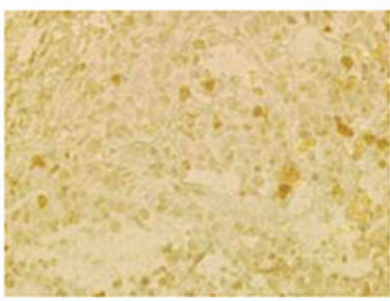

PBS

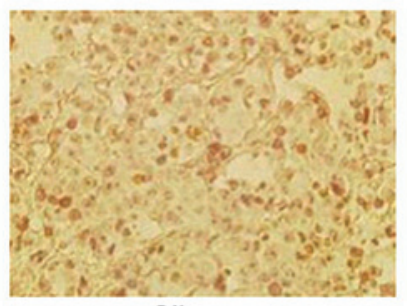

pSilencer

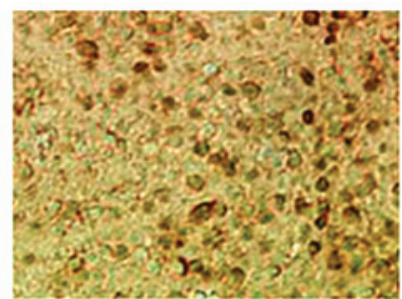

pSilencer-SATB1-shRNA

B

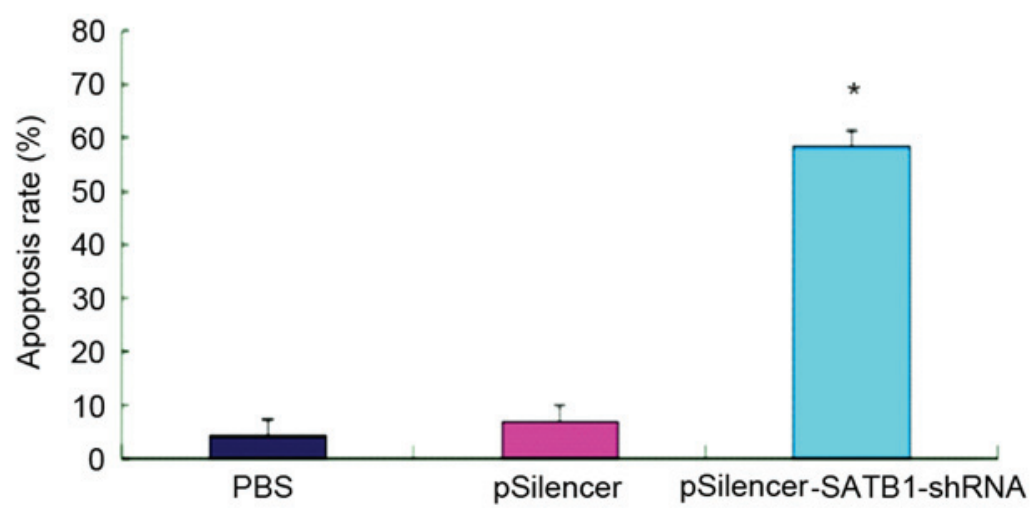

Figure 4. (A) Apoptosis staining using the TUNEL method in tumor tissue samples from each group. (B) Apoptosis rates based on TUNEL results. The pSilencer-SATB1-shRNA vs. pSilencer, or PBS-treated groups ( $\mathrm{P}<0.05, \mathrm{n}=6$ ). SATB1, special AT-rich sequence binding protein 1; sh, short hairpin; pSilencer, plasmid silencer.

pSilencer-SATB1-shRNA inhibits tumor growth. H\&E staining was used to observe the tumor cell growth. In the control (PBS) and empty vector groups (groups A and B), marked tumor growth was observed (Table I). In addition, in 
the two control samples, the cell nuclei were of various sizes and an irregular cell shape was observed (Fig. 3). Conversely, the pSilencer-SATB1-shRNA group demonstrated reduced tumor growth (Table I). In addition, numerous cells had died or were degraded; nuclear pyknosis was observed and normal tissue structures had disappeared (Fig. 3). Together, these results suggested that pSilencer-SATB1-shRNA inhibits tumor growth.

Subsequently, apoptosis in the tumor tissue samples was evaluated using a TUNEL assay (Fig. 4A). The percentage of apoptotic cells in the pSilencer-SATB1-shRNA sample $(58.23 \pm 6.62 \%)$ was significantly increased compared with in the PBS sample $(4.16 \pm 1.83 \%)$ and the empty plasmid sample $(6.84 \pm 3.16 \% ; \mathrm{P}<0.05 ;$ Fig. 4B). Additionally, pSilencer-SATB1-shRNA demonstrated an increased rate of tumor growth inhibition $(83.98 \pm 2.32 \%)$ compared with the controls (Table II).

\section{Discussion}

A prior study on prostate cancer demonstrated that SATB1 staining was more marked if there were metastases compared with if there were not, and that the staining was absent in benign prostate hyperplasia (15). These data suggested that SATB1 serves a crucial role in the metastasis of prostate cancer.

The current study demonstrated that SATB1 protein was produced lower expression in the pSliencer-SATB1-shRNA treatment group compared to pSilencer or PBS-treated groups. Additionally, tumor tissues from the pSilencer-SATB1-shRNA treatment group exhibited reduced tumor growth, with an increased rate of apoptosis, nuclear pyknosis and loss of normal tissue structures. Together, these results indicate that treatment with pSliencer-SATB1-shRNA inhibits xenograft tumor growth in nude mice.

These data are concordant with the results of previous in vivo studies of highly aggressive human prostate cancer cells, which suggested that SATB1 knockdown inhibits tumor growth and invasiveness $(16,17)$. In addition, Mao et al (17) previously examined the effect of a replicative oncolytic adenovirus expressing SATB1-shRNA in a mouse model, and determined that this treatment exhibited a potent antitumor effect against human prostate cancer cells.

In conclusion, the in vivo treatment of prostate cancer with pSilencer-SATB1-shRNA led to a significant reduction in tumor growth and an increased rate of apoptosis. These data demonstrate that pSilencer-SATB1-shRNA is cytotoxic to prostate cancer cells and inhibits tumor growth, indicating their potential as a novel strategy for treating human prostate cancer.

\section{References}

1. Siegel R, Naishadham D and Jemal A: Cancer statistics, 2013. CA Cancer J Clin 63: 11-30, 2013.

2. Shore N: Management of early-stage prostate cancer. Am J Manag Care 20 (12 Suppl): S260-S272, 2014.

3. Gridley DS and Slater JM: Gene therapy: A possible aid to cancer radiotherapy. Discov Med 4: 408-414, 2004.

4. Altaner C: Prodrug cancer gene therapy. Cancer Lett 270: 191-201, 2008

5. Tangney M: Gene therapy for cancer: Dairy bacteria as delivery vectors. Discov Med 10: 195-200, 2010.

6. Singh P, Yam M, Russell PJ and Khatri A: Molecular and traditional chemotherapy: A united front against prostate cancer. Cancer Lett 293: 1-14, 2010.

7. Ambesajir A, Kaushik A, Kaushik JJ and Petros ST: RNA interference: A futuristic tool and its therapeutic applications. Saudi J Biol Sci 19: 395-403, 2012.

8. Bora RS, Gupta D, Mukkur TK and Saini KS: RNA interference therapeutics for cancer: Challenges and opportunities (review). Mol Med Rep 6: 9-15, 2012.

9. Mansoori B, Sandoghchian Shotorbani S and Baradaran B: RNA interference and its role in cancer therapy. Adv Pharm Bull 4: 313-321, 2014.

10. Agrawal N, Dasaradhi PV, Mohmmed A, Malhotra P, Bhatnagar RK and Mukherjee SK: RNA interference: Biology, mechanism, and applications. Microbiol Mol Biol Rev 67: 657-685, 2003.

11. Lee Y, Ahn C, Han J, Choi H, Kim J, Yim J, Lee J, Provost P, Rådmark O, Kim S and Kim VN: The nuclear RNase III Drosha initiates microRNA processing. Nature 425: 415-419, 2003.

12. Kohwi-Shigematsu T, Poterlowicz K, Ordinario E, Han HJ, Botchkarev VA and Kohwi Y: Genome organizing function of SATB1 in tumor progression. Semin Cancer Biol 23: 72-79, 2013.

13. Yasui D, Miyano M, Cai S, Varga-Weisz $P$ and Kohwi-Shigematsu T: SATB1 targets chromatin remodelling to regulate genes over long distances. Nature 419: 641-645, 2002.

14. Han HJ, Russo J, Kohwi Y and Kohwi-Shigematsu T: SATB1 reprogrammes gene expression to promote breast tumour growth and metastasis. Nature 452: 187-193, 2008.

15. Mao L, Yang C, Wang J, Li W, Wen R, Chen J and Zheng J: SATB1 is overexpressed in metastatic prostate cancer and promotes prostate cancer cell growth and invasion. J Transl Med 11: 111, 2013.

16. Shukla S, Sharma H, Abbas A, MacLennan GT, Fu P, Danielpour D and Gupta S: Upregulation of SATB1 is associated with prostate cancer aggressiveness and disease progression. PLoS One 8: e53527, 2013.

17. Mao LJ, Zhang J, Liu N, Fan L, Yang DR, Xue BX, Shan YX and Zheng JN: Oncolytic virus carrying shRNA targeting SATB1 inhibits prostate cancer growth and metastasis. Tumour Biol 36: 9073-9081, 2015. 